\title{
EGR1 Regulates the Thickning of The Peroneal Tendon in Taekwondo Athletes by Activating the STAT3 Pathway, Thereby Increasing the Risk of Dislocation
}

\section{Liqiang Wang}

department oforthopedics $₫$ the China-japan friendship hospital

\section{Bohan Li}

The Fourth Affiliated Hospital of Hebei Medical University

Zhiyu Zhang ( $\nabla$ xiangyubo2020@126.com )

Fourth Affiliated Hospital of China Medical University https://orcid.org/0000-0002-5500-9593

\section{Fusheng Yuan}

The Fourth Affiliated Hospital of Hebei Medical University https://orcid.org/0000-0002-7101-0877

\section{Research Article}

Keywords: Peroneal tendon dislocation, Taekwondo athletes, EGR1, IL-20, Traditional Chinese Medicine Orthopedics.

Posted Date: February 15th, 2022

DOI: https://doi.org/10.21203/rs.3.rs-1338991/v1

License: (9) This work is licensed under a Creative Commons Attribution 4.0 International License. Read Full License 


\section{Abstract \\ Background}

Taekwondo is a very popular sport today. Due to higher training intensity, ankle instability and PTD are common among Taekwondo athletes which may ruin their careers. However, the risk factors and mechanisms of the disease, appropriate treatment methods or preventive measures have not been systematically studied, and this research aims to solve these problems.

\section{Methods}

A large number of Taekwondo athletes have undergone long-term specialist examinations, treatments and postoperative follow-ups. Intraoperative observation and imaging evidence confirmed that the width of the peroneal tendon of Taekwondo athletes was significantly higher than that of the general population, which is one of the cause of PTD.While giving to athletes surgical treatment(multiple reviet technique), we obtained the extraneous tissue in the thickened part of the peroneal tendon from some athletes for follow-up experimental research.

\section{Results}

We found that the concentration of IL-20 in the matrix is relative higher in the thickened peroneal tendon, and it is positively correlated with the expression of EGR1 in fibroblasts, while the dysregulation of EGR1 will activate STAT3 Pathway, and regulate the thickening of the peroneal tendon in a variety of ways.Based on the professional characteristics of athletes, the high incidence of PTD among Taekwondo athletes makes it more important to prevent it. According to the experience of Chinese orthopedics, Astragalus polysaccharide is creatively used as a preventive drug to some athletes who have a high risk of PTD, it was found that the use of APS had a satisfactory effect on delaying the thickening of the peroneal tendon of athletes and reducing the incidence of dislocation. At the same time, the preventive effect of APS was confirmed through several groups of clinical cases, and the mechanism of APS to prevent PTD was also speculated.

\section{Conclusions}

EGR1 regulates the thickning of the peroneal tendon in Taekwondo athletes by activating the STAT3 pathway, the importance of prophylactic useof APS to reduce the thickening of the peroneal tendon.

\section{Introduction}

Taekwondo originated on the Korean Peninsula and is one of the official competitions of the modern Olympic Games[1, 2]. There are between 75 and 120 million practitioners of Taekwondo in more than 140 
countries in the world. Taekwondo athletes attack their opponents using forearm and leg movements; leg movements account for about $70 \%$ of Taekwondo technical movements. Although Taekwondo can improve physical and psychological conditions, its tendency to cause movement-related injuries cannot be ignored. Sprains, soft tissue injury and joint damage are the most common types of injury[3]. There are relatively complete epidemiological data on injuries in Taekwondo competitions; unfortunately, there are few reports on the acute, chronic and long-term injuries of non-entrant athletes during training. Among these injuries, ankle instability and dislocation of the peroneal tendon are very common among Taekwondo athletes. The occurrence of PTD is a fatal blow to Taekwondo athletes and may ruin an athlete's career.

However, the risk factors of the disease and appropriate treatment methods have not been systematically studied.An abnormality of the peroneal tendon may affect the stability of the ankle joint $[4,5]$. Injuries of the superior peroneal retinaculum may lead to dislocation of the peroneal tendon, and injuries of the anterior tibiofibular ligament may result in chronic ankle instability [6, 7]. At present, the exploration of the causes of PTD is still in progress. The known injury mechanisms are: $₫$ malleolar sulcus dysplasia, relaxation or absence of the superior fibular retinaculum, $\nabla$ sports injury, $\nabla$ malunion after lateral malleolus fracture and deformity of dorsiflexion and valgus foot can also cause fibular tendon slippage, $\mathbb{Q}$ Neuromuscular diseases (such as poliomyelitis and cerebral palsy) are often accompanied by fibular tendon dislocation[8]. However, for Taekwondo athletes, the occurrence of PTD is mostly due to sports injuries. After a large number of long-term specialist examinations and follow-up of Taekwondo athletes, we found that there are various relationships among athletes with PTD, such as age, sex, training duration(years) and daily training duration, as well as peroneal tendon width, which we pay special attention to because of the intraoperative observation and imaging evidence. The thickening of the peroneal tendon may be related to the athletes' individual training intensity, fixed training technical movements, etc. Therefore, we have studied whether the thickening of peroneal tendon will increase the incidence of PTD, and the result is positive. While peroneal tendon thickening is probably one of the most important causes of PTD in athletes.

To understand the thickening mechanism of the peroneal tendon, it is necessary to understand its internal structure and function: Tendons are the connective tissue fibers at the end of muscles that help the muscles attach to bones or other structures. Tendons are tougher and smaller than muscles and are mainly composed of parallel collagen fiber bundles and have no contraction ability[9]. The tendon is composed of a large number of collagen fibers and a small amount of matrix and fibroblasts between the collagen fibers[10], so it is obvious that the regulation of fibroblasts, cytokines and inflammatory factors in the matrix on the thickening of the tendon cannot be ignored. The matrix plays a key role in the power transmission of tissues and the maintenance of tendons, ligaments, bones and muscles and the structure of the tissues[11]. High-intensity exercise will significantly affect the renewal of matrix components, collagen synthesis and metalloproteinase activity. The expression and release of inflammatory factors in local tissues are enhanced after exercise [12]. These changes are likely to have a significant impact on the thickening of the peroneal tendon. Based on this hint, we carried out follow-up experiments to prove the above speculation. Understanding the cause of the disease is the first step for treating it.Currently, the 
clinical treatment of PTD is mostly based on surgical treatment: for example, fibular groove deepening, fibular osteotomy, tissue graft repair and peroneal tendon re-routing [9]. In view of the fact that Taekwondo athletes need a highly stable ankle joint, the above methods have inherent limitations, and surgical treatment has more or less adverse effects on them, such as shortening their careers and reducing their athletic ability. However, drug therapy and preventive treatment have not yet been extensively studied.

Drug prevention of PTD may sound like a myth at first, but this study shows that it may has many advantages in extending the career and protecting the athletic ability of taekwondo athletes and other individuals with a high demand for ankle stability. Although the diagnosis and treatment methods of western medicine have always been in a dominant position in the treatment of bone injuries, the Traditional Chinese Medicine Orthopedics has a history of thousands of years in China. It has its own traditional and incisive manual treatment combined with traditional Chinese medicine. These concepts of non-surgical treatment of Traditional Chinese Medicine Orthopedics have sometimes been applied to our clinic, helping us solve many clinical problems. Therefore, we firstly identified some athletes as being at high risk for PTD based on their peroneal tendon thickening degree, according to the experience of Traditional Chinese Osteology, then administered APS as prophylactic drug, and so far, the athletes in the experimental group got satisfactory results. In the future, more and more attention will be paid to the preventive treatment of PTD, which will provide protection for more Taekwondo athletes and other similar groups.

\section{Materials And Methods}

Demography: We collaborated with the provincial Taekwondo sports team. During 2012-2017, we surveyed a total of 178 athletes from the provincial Taekwondo team. We conducted a questionnaire survey along with annual physical and biomedical imaging examinations on the athletes each year. A total of 85 athletes at high risk of PTD were selected from 178 athletes, and this group was called the high-risk group. (high risk group: athletes with a peroneal tendon width greater than $7.00 \mathrm{~mm}$ (1.25*normal width) were assumed to be athletes with thickened peroneal tendon and may have a high risk of dislocation.). At the same time, to measure the normal value of peroneal tendon width in nonathletes, we investigated MRI results in 220 non-athletes from our patients.

\section{Bone tendon tissue source}

Peroneal tendon tissue was collected from 27 patients with PTD from 2012 to 2017 with partial thickening of the peroneal tendon. Take 3specimens from the high-risk group and 3specimens from the low-risk group. After obtaining the informed consent of 27 participants, a biopsy was performed. That is, the protein of tendon fibroblasts is extracted and the cytokines and inflammatory factors in the matrix are detected.

\section{Isolation and culture of primary fibroblasts}


Strictly follow the aseptic operation, soak the surgical specimens in $0.05 \%$ chlorhexidine solution for 5 minutes, and rinse with $0.9 \%$ sodium chloride solution for 3 times; place in $0.25 \%$ pancreatin at $4^{\circ} \mathrm{C}$ Cold digest for 18 hours, wash with DMEM, fully cut the tendons, add $0.25 \%$ type I collagenase, incubate at $37^{\circ} \mathrm{C}$ for 1 hour, pipette and cut the tissue, filter the digestion solution, collect the cell suspension, centrifuge at $1000 \mathrm{rpm}$ for 5 minutes, discard The supernatant is a cell suspension cultured in DMEM containing $10 \%$ fetal bovine serum, and the cell density is adjusted to $5^{\star} 105 / \mathrm{ml}$, inoculated into a culture flask, and cultured in an incubator at $37^{\circ} \mathrm{C}$ and $5 \% \mathrm{CO} 2$. Replace the culture medium once in 2-3 days. The 4-5th passage cells were used in this study.

\section{Main experimental reagents}

DMEM culture medium (Shanghai Tuoyang Biotechnology Co., Ltd.), Fetal Bovine Serum (Zhejiang Tianhang Biotechnology Co., Ltd.), 0.25\% trypsin (Shanghai Gooduo Biotechnology Co., Ltd.), IL-20 (USA PEPROTECH EC company), IL-20 ELISA test kit, IL-6 ELISA test kit, CSF ELISA test kit, TNF-a ELISA test kit, EGF ELISA test kit, IFN ELISA test kit, TGF- $\beta$ ELISA Detection kit (Wuhan Boster Bioengineering Co., Ltd.). SYBR PreMix ex Taq II kit (Takara). Anti-GAPDH (Cell signaling technology), anti-ß-actin (Cell signaling technology), anti-EGR1 (Cell signaling technology), anti-P-STAT3 (Cell signaling technology), anti-STAT (Cell signaling technology)), all secondary antibodies (zsgb-Bio) .

\section{Inflammatory cytokine detection in tendon extracellular matrix}

Strictly follow aseptic operation, soak the specimens obtained from the operation with $0.05 \%$ chlorhexidine solution for 5 minutes, and rinse with $0.9 \%$ sodium chloride solution for 3 times; place in $0.25 \%$ pancreatin, Cold digestion at $4^{\circ} \mathrm{C}$ for 18 hours, wash with DMEM, fully cut the tendons, blow and cut the tissue with a pipette, centrifuge at $1000 \mathrm{rpm}$ for 5 minutes, take the supernatant, and use an ELISA test kit to determine IL-20, IL-6, CSF, TNF-a, EGF, IFN, TGF- $\beta$, etc.

\section{Real-time reverse transcription polymerase chain reaction (RT-PCR)}

Use MiniBEST universal RNA extraction kit (Takara, Shiga, Japan) to extract total RNA for real-time quantitative PCR detection. Primescript RT master Mix (Takara) was used for reverse transcription. Realtime PCR was detected using LightCycler 480 real-time PCR system (Roche) and SYBR PreMix ex Taq II kit (Takara). Under general amplification conditions, the IL-20, EGR1, and STAT3 listed in Table 1 were amplified. The experimental data uses the $2-\triangle \Delta C T$ method to determine the relative multiples of mRNA expression changes.

Western blot analysis: After treatment, the cells were washed with ice-cold PBS and placed in a lysis buffer containing a cocktail of protease and phosphorylase inhibitors $(50 \mathrm{mM}$ Tris- $\mathrm{HCl}, 150 \mathrm{mM} \mathrm{NaCl}, 1 \%$ NP-40, $0.5 \%$ deoxycholic acid). Sodium, $0.1 \%$ sodium lauryl sulfate). After centrifugation at $12000 \mathrm{~g}$ for $30 \mathrm{~min}$ at $4^{\circ} \mathrm{C}$, the supernatant was used to collect protein containing total protein. The protein concentration was determined with the BCA protein concentration determination kit (Boster, Hubei, China). The samples were separated by SDS-PAGE, and then transferred to a polyvinylidene fluoride 
(PVDF) membrane (Millikon, Massachusetts, USA) (210 mA, 30-120 min depending on the molecular weight of the protein). After blocking the PVDF membrane with $5 \%$ skim milk for $2 \mathrm{~h}$, incubate overnight at $4^{\circ} \mathrm{C}$ with a suitable primary antibody (diluted $1: 500$ or 1:1000). Then, the blot was incubated with antimouse or anti-rabbit IgG conjugated with horseradish peroxidase (1:5000 dilution) at room temperature for $1 \mathrm{~h}$. An EC3 imaging system (UVP Inc., California, USA) was used to display the immunoreactive bands, and the optical density of each band was measured with ImageJ software (NIH, USA). In these experiments, GAPDH and $\beta$-actin were used as whole cell protein load controls. The ratio between the protein of interest and the load control of the same sample is calculated as the relative content and represented graphically. The results are the average of three independent experiments.

\section{The effect of IL-20 on the expression of EGR1 in fibroblasts}

According to different risk factors, the cultured fibroblasts were divided into $\mathrm{H} 1$ high-risk group and L1 low-risk group. Divide the 24-well culture plate into 3 groups, take 8 wells from each group, and add 5*103 cells to each well. Incubate in an incubator at $37^{\circ} \mathrm{C}$ and $5 \% \mathrm{CO} 2$ for 12 hours, and then aspirate and discard the culture solution.. According to the different concentrations of IL-20 added, the L1 group was marked as F0,1 and 3 groups. Each well was added with $600 \mathrm{uL}$ of (IL-20 containing $0 \mathrm{U} / \mathrm{ml}, 1 \mathrm{U} / \mathrm{ml}, 3 \mathrm{U} / \mathrm{ml})$ DMEM culture medium respectively. The expression of EGR1 in each group of cells was detected on 0,12 , 24,48 and 72 hours.

Animal experiment: clean adult male Sprague Dawley (SD) rats, weight: 110-150 g, purchased from Beijing Huafukang Biotechnology Co., Ltd. The company has been approved by the International Animal Control Committee (IACUC). We make every effort to minimize the suffering of animals during our experiments, and we try to reduce the number of animals used as much as possible. A total of 60 rats were used in our study. And the experimental results obtained include all experimental animals, without excluding any set of data. Before the start of the experiment, the rats were randomly divided into groups. The personnel responsible for allocating and conducting the experiment are different from the personnel evaluating the results. In the experimental group treated with IL-20 (20 in each experimental group, 40 in total), a $5 \mathrm{ml}$ syringe was used to inject $5 \mathrm{ml}$ of recombinant rat IL-20 protein into the Achilles tendon of both hind limbs of the rat, with the concentrations of $0.2 \mathrm{U} / \mathrm{ml}$ and $0.6 \mathrm{U} / \mathrm{ml}$ (Defined the concentration of IL-20 in the matrix of the peroneal tendon tissue in the L1 group as 1 unit concentration), the control group (20 rats) used the same method to inject an equal volume of saline. The rats were injected 3 times a week for 1 weeks. After 6 weeks, the rats were sacrificed. The Achilles tendons of the right hind limbs of the sacrificed animals were immediately stored in liquid nitrogen or fixed with $4 \%$ paraformaldehyde solution for experimental analysis.

Statistical analysis: All data obtained in the experiment were analyzed using the software program GraphPad Prism 6.02. The difference between the two groups was statistically significant, and the test was used. Quantitative variables are expressed as mean \pm standard deviation (SD). One-way analysis of variance (ANOVA) was used to compare multiple groups. $P$ value $<0.05$ is considered statistically significant. 


\section{Result}

\section{Risk factors of PTD}

Based on intraoperative findings, clinical follow-up and data analysis, we have launched an exploration of PTD in Taekwondo athletes.

\subsection{General data analysis of athletes}

Statistical analysis, based on the distribution of cases by gender, age, affected leg, training year, annual average training days and daily average training time. The results indicated that most of the patients were male. However, by considering the gender ratio of the recruited patients, it was found that the incidence of PTD in females (especially in women with less developed muscles ) was significantly higher than that in males. The onset time of the injury was concentrated within the first year of training or at over 5 years of training, and the right ankle was the one more commonly affected,and we also found in a long-term questionnaire survey and inquiries that athletes' PTD is mainly related to their training intensity of their preferred foot and muscle strength around the peroneal tendon. (Table 1) 
Table 1

Patient demography and training-related data (mean and standard deviation).

\begin{tabular}{|ll|}
\hline Patients(n=27) & \\
\hline Age(years) & $18.7(3.9)$ \\
\hline Sex(n) & \\
\hline Female & 11 \\
\hline Male & 16 \\
\hline Incidence rate by sex(\%) & \\
\hline Female & 19.6 \\
\hline Male & 13.1 \\
\hline Affected foot(n) & \\
\hline Right & 21 \\
\hline Left & 6 \\
\hline Training duration(years) & 8 \\
\hline$<1$ & 2 \\
\hline $1-3$ & 5 \\
\hline $3-5$ & 12 \\
\hline$>5$ & $287.4(32.5)$ \\
\hline Average number of training days per year(days) & $8.6(1.8)$ \\
\hline Average daily training hours(h) & \\
\hline
\end{tabular}

\subsection{Intraoperative discovery}

New technique called multi-rivet technique for peroneal retinaculum reconstruction was used in surgery and got better result in athletes. As shown in the pictures during the operation(figure1-A), when 27 athletes were treated with surgery, we found that most athletes had varying degree of thickening of the peroneal tendon, which resulted in a relatively shallow fibular groove and easily slipped away.This commonality may indicate that the thickening of the peroneal tendon is an important cause of PTD in athletes.

\subsection{Thickening of the peroneal tendon is a major cause of PTD}


A normal peroneal tendon is $5.63 \pm 0.69 \mathrm{~mm}$ wide(we performed MRI examination on 220 non-athlete patients, and measured the width of peroneal tendon to obtain the mean and standard deviation).

The results of the width of the peroneal tendon were obtained from the MRI of all taekwondo athletes surveyed each year in 2012-2017, (Figure 1-B) shows that the thickened peroneal tendon of athlete in high-risk group is $7.81 \mathrm{~mm}$ wide and that in normal peroneal tendon in non-atheles group is $5.77 \mathrm{~mm}$ wide,therefore, as a large number of MRI results show, there are varying degrees of thickening of the peroneal tendon in Taekwondo athletes.

From 2012 to 2017, the annual incidence of PTD among all Taekwondo athletes and the annual incidence of PTD among athletes with thickened peroneal tendon were shown in (Figure 1-C). The two curves show that the dislocation rate of the high-risk group with thickened peroneal tendon was significantly higher than that of all athletes group,while The reason for the year-over-year decrease in the incidence of PTD in both groups was that athletes in the high-risk group of PTD mentioned in the following study used APS as a prophylactic drug in 2014. However, the results do not affect the results of this group of experiments.

In summary, we speculate that Taekwondo athletes may gradually thicken their peroneal tendons due to year-round overload training and other reasons like injury. Thickened peroneal tendons and relatively shallow peroneal grooves are important causes of Taekwondo athletes' PTD. Therefore, in order to control the thickening peroneal tendon, we conducted the following experiments to explore the related mechanism of peroneal tendon thickening.

\section{The Mechanism Of Peroneal Tendon Thickening}

Tendon is composed of a large number of collagen fibers, a small amount of matrix and fibroblasts between collagen fibers. Although the number of collagen fibers is much greater than fibroblasts and the matrix, The regulatory of fibroblasts and the cytokines and inflammatory factors in the matrix cannot be ignored. Based on the results of the etiological exploration, we predict that the local aseptic inflammation caused by the overloaded exercise and sports injury of Taekwondo athletes will inevitably lead to the participation of Inflammatory cytokines, and then affect the composition and structure of the peroneal tendon. Based on this speculation, we carried out the follow-up experiments.

During the operation, we obtained the excess resection tissue of the thickened peroneal tendon of 27 athletes, and removed 3 tissues from the high-risk group that were significantly thickened (width is greater than $7.00 \mathrm{~mm}$ ) and 3 tissues from the low-risk group(less than $7.00 \mathrm{~mm}$ ), and defined them as $\mathrm{H} 1$ H3 group tissues, L1-L3 group tissues, isolate matrix for follow-up experiments(H1-H3/L1-L3 group matrix) and isolate fibroblasts in tissues and primary culture for cell lines(H1-H3/L1-L3 cell lines)

\subsection{Abnormalities of fibroblasts and matrix}


Known from the sample tissues pairs obtained: the differential expression of mRNA and protein in fibroblasts between the high-risk and low-risk groups, and the difference in the concentration of cytokines and inflammatory factors in the matrix.

\subsubsection{The thickening of peroneal tendon may be related to the expression of EGR1 in tendon.}

We performed protein extraction and protein quantification on all the above-mentioned clinically obtained tissue pairs .Among the many kinds of differentially expressed proteins between high-risk and low-risk group, We found that the top 7 proteins differentially expressed in the two groups are:EGR1,C-

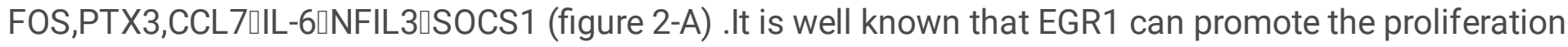
and differentiation of tendon cells, stimulate collagen protein and EGR1 also closely related to inflammation[13]. So EGR1 may play a role in the thickening of the peroneal tendon, which has attracted our attention.. The expression level of EGR1 in the cell lines in the high-risk group was significantly higher than low-risk group. That means the thickening of the peroneal tendon may be related to the expression of EGR1 in the tendon. (figure 2-B)

\subsubsection{The high expression of EGR1 is mainly may due to its high expression in fibroblasts.}

In order to explore whether EGR1 is highly expressed in collagen, matrix or fibroblasts, we performed WB and qrtPCR experiments in each H1-H3/L1-L3 cell line and specifically test the mRNA and protein levels of EGR1 in fibroblasts. Experiments have confirmed that in fibroblasts isolated from tissues removed from athletes with PTD, EGR1 is highly expressed in protein(figure 2-C) and mRNA(figure 2-E) levels. And the differential expression in tissues and simple fibroblasts is almost the same(figure 2-D),so we have reason to believe that the high expression of EGR1 in peroneal tendon is mainly may due to the high expression in fibroblasts.

\subsubsection{In high-risk group:the concentration of some inflammatory cytokines in the matrix increases differently}

The Elisa kit was used to determine the inflammatory cytokines in the matrix with increased differently in the high-risk and lower-risk groups. For the test results of inflammatory cytokines, the concentration of IL,IFN,EGF,CSF,TNF-a,TGF- $\beta$ in the matrix changes significantly(figure2-F). The inflammatory cytokines with different changes in the matrix may have an effect on the thickening of the peroneal tendon. For example, interferon may affect the redistribution of collagen in the peroneal tendon by changing the immune microenvironment around the tendon and other complex ways; EGF can regulate cell growth and other cell functions by binding to specific, high-affinity cell membrane receptors which may also affect the state of fibroblasts and thus the width of the peroneal tendon; IL family may affect the peroneal tendon thickening by affecting the progression of inflammation around the peroneal tendon, The role of IL family in inflammatory response has been widely reported, so IL has attracted our attention,However, 
the specific mechanism is not yet known. Since IL-20 is related to the control of local inflammation and fibrosis effects[14], we chose IL-20 for targeted research.(figure 2-F)

In summary, the expression of EGR1 in fibroblasts in the high-risk group increased significantly(figure 2$\mathrm{E})$; the concentration of IL-20 in the matrix increased significantly in the high-risk group(figure 2-F).Based on the above findings, we guessed that there may be a correlation between the two conclusions(IL-20 is located in the internal environment outside fibroblasts, and its concentration changes may cause a cascade reaction in fibroblasts, which in turn leads to fibroblast biology behavior changes, resulting in changes in the width of the peroneal tendon), so we conducted follow-up experiments.

\subsection{The concentration of IL-20 in exogenous extracellular matrix is positively correlated with the expression of EGR1 in fibroblasts}

Obtain the primary cultured L1 group cell line (fibroblasts), divide them into 3 equal parts and culture them for $72 \mathrm{~h}$ at 0 unit, 1 unit and 3 unit concentration(defined them as F0/F1/F3group) (Defined the concentration of IL-20 in the matrix of the peroneal tendon tissue in the L1 group as 1 unit concentration).

The F0/F1/F3 groups of cells obtained were subjected to qRTPCR experiments to specifically detect the expression of EGR1 at the mRNA level. As an example, the expression level of EGR1in F3 group was measured at $0 \mathrm{~h}, 12 \mathrm{~h}, 24 \mathrm{~h}, 48 \mathrm{~h}$, and $72 \mathrm{~h}$ respectively.We found that within a certain range, the higher the concentration of IL-20 in the matrix, the more expression of EGR1 in fibroblasts in the thickened peroneal tendon(figure3-A); and since the only variable is controlled, we can conclude that the exogenous inflammatory cytokine IL-20 in the extracellular matrix of fibroblasts leads to (or indirectly leads to)the differentially high expression of EGR1 in fibroblasts.(figure 3-A).

\subsection{The high expression of EGR1 regulates the thickening of the peroneal tendon by targeting the activation of the STAT3 pathway}

In order to explore how the overexpression of EGR1 in fibroblasts affects the thickening of the peroneal tendon, which leads to the PTD of Taekwondo athletes, we conducted the following investigation.According to many previous reports, the activation of the STAT3 pathway is related to the morphological changes of the tendon. The activation of STAT3 promotes the transition from fibroblasts to myofibroblasts, collagen release and fibrosis in vitro and in vivo, which is verified in other diseases such as systemic sclerosis and tendon injuries.So we speculate that it might be the activation of the STAT3 pathway in the peroneal tendon of athletes that leads to the decrease of fibroblasts, the release and redistribution of collagen, and the fibrosis of collagen, which leads to changes in the width of the peroneal tendon. So we tried to verify whether the high expression of EGR1 can activate the STAT3 pathway, and thus regulate the thickening of the peroneal tendon through the STAT3 pathway. We conducted a WB experiment and concluded that the high expression of EGR1 activates the STAT3 pathway(figure3-B), and the up-regulated STAT3 then has the above-mentioned effect on the width of the peroneal tendon. The above speculation was confirmed. 


\subsection{Retrospective verification in animal experiments}

Injecting $5 \mathrm{ml} \mathrm{IL-20}$ of 0, 1 and 3concentration into the Sprague Dawley tendons of 3 mice(define them as mice 1,2,3).45 days later, the bilateral Achilles tendons of the SD were taken out, and we measured these Achilles tendons separately:the relative width of EGR1 (figure3-C) and the relative expression of EGR1 (figure3-D) of the mRNA level in the Achilles tendons tissues. According to the results, it was found that after 45 days, within a certain concentration range, the higher the concentration of IL-20 locally injected into the Achilles tendon, the wider the width of the Achilles tendon, and the higher the expression of EGR1 in the tissues. Both are positively correlated, which is consistent with the previous conclusion. However, due to the complex mechanism of IL-20 in vivo, this animal experiment is only a retrospective verification. But satisfactory results were obtained.

To sum up all the exploration of the thickening of the peroneal tendon of Taekwondo athletes: It may be due to the chronic injury and aseptic inflammation of the peroneal tendon caused by the professional characteristics of athletes, which in turn increases the concentration of IL-20 in the matrix of peroneal tendon. However, it is precisely because of the increase in the concentration of IL-20 around fibroblasts that, through a complex method, the expression of EGR1 in fibroblasts is significantly high at the mRNA level, which in turn activates the STAT3 pathway and promotes the transition from fibroblasts to myofibroblasts, collagen release, and fibrosis in vitro and in vivo. The accumulation of collagen and the increase in fibrosis lead to the thickening of the peroneal tendon finally.

\section{Preventive Treatment Of Peroneal Tendon Thickening}

\subsection{Astragalus polysaccharide as a preventive medication for PTD}

Based on the experience of traditional Chinese medicine in the treatment of orthopedic diseases, since the beginning of 2014, we have given regular astragalus polysaccharide (APS) as a prophylactic drug to athletes(85) in the high-risk group of dislocation. We found that the average width of the peroneal tendon increased at a slower rate in this group.And after 2-3 years of medication, the peroneal tendon of some athletes stopped thickening and even realized negative growth tending to the low-risk value (Figure 4A);the incidence of PTD in this group of athletes decreased year by year. These two results, which were carried out over a period of 6 years, suggest that APS as a prophylactic drug has a significant effect on reducing the incidence of PTD in taekwondo athletes.(Figure 4-B). This result proves that in the special profession of Taekwondo athletes, preventive and targeted use of traditional Chinese medicine,Astragalus polysaccharides, with low side effects and slow pharmacological effects can counteract the mechanical damage caused by overload exercise and reduce the incidence of PTD has an role cannot be ignored.

\subsection{Several clinical cases as evidence of the effectiveness of preventive use of APS}


In the long-term clinical follow-up, we found that 7 patients in the high-risk group(among 85 athletes) who had preventively used APS for more than 2 years but undergo the PTD surgery. During the operation, we obtained the excess resection tissue of the thickened peroneal tendon of 7 athletes, (defined them as M17groups)and isolate the fibroblasts and matrix from tissues.

It can be concluded that the average width of the peroneal tendon in the $M 1,2,3,4,5,6,7$ group( $M$ group) is similar to the average of the $L 1,2,3,4$ group( $L$ group), and is also less than $7.00 \mathrm{~mm}(6.93415)$ (figure4-C); the average concentration of IL-20 in the peroneal tendon matrix in the $\mathrm{M}$ group is also less than the average concentration of the $L$ group(figure4-D); while the average expression of EGR1 in the fibroblasts in $\mathrm{M}$ group is similar to the average expression of the $\mathrm{L}$ group. (figure4-E). The above conclusions reflect that preventive use of APS in Taekwondo is necessary to delay the increase in the width of the peroneal tendon and reduce the incidence of PTD.

\section{Discussion}

In the current study, the incidence of dislocation of the peroneal tendon in Taekwondo athletes in our province was as high as $15.2 \%$, in the 178 Taekwondo athletes we surveyed, the incidence of PTD was $17.8 \%$ in 2012-2013 (before prophylactic intervention). In the literature, there are reports that Taekwondo athletes had a higher incidence of dislocation of the tendon did than the general population[1,3].The incidence of PTD in female athletes was higher than that in males, suggesting that female athletes are more prone to ankle injury. This could be due to less advanced Taekwondo skill development in the females studied compared with the males, or because the ankle ligaments of women are thinner and less protected than those of men. The high incidence of tendon dislocation among novice trainees again suggested that the proficiency of technical movements affects the incidence of ankle injuries. However, a high incidence in experienced athletes suggested that long-term high-intensity training could lead to gradually deteriorating stability of the ankle joint, eventually resulting in injury. In addition, the majority of patients had right ankle injuries. This could be related to the dominant technical movement of the Taekwondo side kick using the right leg. But more importantly, intraoperative observation and MRI results showed that the peroneal tendon of experienced Taekwondo athletes was significantly wider than that of the general population. We postulate that this is related to the repeated training of professional movements, including the taekwondo side kick. The side kick is one of the most important movements in Taekwondo. When the movement is completed, the ankle joint is extremely dorsiflexed, and the peroneus longus and brevis muscles are in a state of loaded pulling. When performed repeatedly over a long period, this causes the peroneal tendon to be thickened, which makes the fibular groove relatively shallow and causes the superior peroneal retinaculum to be under stress. Such conditions in turn lead to relaxation and rupture of the superior peroneal retinaculum and increase the probability of dislocation of the peroneal tendon.

The above assumptions may explain the high incidence of dislocation of the thickened peroneal tendon in Taekwondo athletes. In other words, the thickening of the peroneal tendon is an important risk factor for PTD, Therefore, in our experimental results, we found that most patients with PTD are mostly patients 
with thickening of the peroneal muscle in the high-risk group. They have severely thickened the peroneal tendon due to long-term training, which makes the peroneal groove relatively shallow, which eventually leads to dislocation of the peroneal tendon. This research aim to reveal the related mechanisms of peroneal tendon thickening and provide a guidance of preventive methods, thereby reducing the incidence of PTD in this special athletes group.

The exploration of the mechanism began with the in situ resection of excess tissue from 8 athletes with thickening of the peroneal tendon. Through experiments, we have obtained multiple differentially expressed genes in fibroblasts and inflammatory cytokines with different concentrations in multiple substrates between the high-risk and low-risk groups of peroneal tendon thickening. Among the many differential genes in fibroblasts of thickened peroneal tendon, EGR1 has its own role in regulating the strength, morphology and fibrosis degree of the tendon [15], so we have a strong interest in EGR1. IL-20 is a soluble protein that belongs to the IL-10 cytokine family. And it is secreted by activated keratinocytes and monocytes, and transmits signals into the cell through two receptors expressed on the surface of other endothelial cells. It plays an important role in controlling inflammation, especially in regulating skin inflammation and aseptic inflammation of skeletal muscle[16]. Therefore, the up-regulation of IL-20 concentration in the matrix of the thickened peroneal tendon (also because there is no previous report) has also attracted our attention.After subsequent experiments, we found that within a certain range, the higher the concentration of exogenous IL-20 around fibroblasts, the higher the expression of EGR1 in fibroblasts. This positive correlation may represent: due to the professional characteristics of athletes, overloading exercise and fatigue injury lead to chronic aseptic inflammation of the peroneal tendon, which in turn leads to an increase in IL-20 concentration. The increase in IL-20 concentration leads to the high expression of EGR1 in fibroblasts and the subsequent cascade reaction, which in turn affects the width of the peroneal tendon. To explore this issue, we tried to link the EGR1 and STAT3 pathway, and finally confirmed that the high expression of EGR1 activates the STAT3 pathway, which in turn promotes the transition from fibroblasts to myofibroblasts, collagen release, and fibrosis in vitro and in vivo[17], this leads to the increase and redistribution of collagen in the peroneal tendon, as well as the increase of fibrotic material, which eventually causes the athlete's peroneal tendon to slowly thicken.

As for the treatment, surgical treatment is still the first choice after the occurrence of PTD in Taekwondo athletes. Although we did not describe the related problems of the surgical method in detail in the text, we have a lot of findings and tips in the surgical treatment of these 27 athletes with PTD. So we have a deeper understanding of this disease because of the process of surgical treatment. There is currently a range of surgical treatment options available. The objective of fibular groove deepening is to increase the stability of the peroneal tendon by deepening part of the posterior side of the distal radius of the fibula[18]. We did not employ this surgical plan. For athletes with thickening of the peroneal tendon, fibular groove deepening cannot achieve the satisfactory tendon anchoring effect within the limited width of the peroneal tendon. Moreover, excessive removal of the bone may reduce the strength of the lateral wall of the fibular groove. This would present a potential risk of stress fracture when bone strength is reduced and muscle tendon pressure is increased; the remodeled bone structure also has an issue of lower strength. Although it is possible to confirm visually that the reconstructed bone covers the tendon 
during surgery, the strength of the reconstructed bone structure is not predictable in post-operative rehabilitation training, especially for the high-intensity training undertaken by Taekwondo athletes.Jones et al. described a method of moving the distal end of the Achilles tendon at the tip of the fibula to strengthen the peroneal retinaculum and thus stabilize the peroneal tendon. The fibularis brevis, the fascia and the Achilles tendon[19] may also strengthen the retinaculum. In fact, many surgical methods can be used to reconstruct the tendon[20], although most of them use the calcaneofibular ligament to stabilize the peroneal tendon. For injured athletes, we did not adopt the soft tissue graft repair method nor the peroneal tendon re-routing method. In the case of a defined fracture of the superior peroneal retinaculum, the strength and function of the injured ligament would be limited by the soft tissue graft repair method. In addition, iatrogenic damage to the patient's posterior malleolus may result during harvesting of the soft tissue. This is not advisable for Taekwondo athletes, who require extremely high stability of the fibula. Similarlyadvisable for Taekwondo athletes, who require extremely high stability of the fibula. Similarly the tendon re-routing method leads to alteration of the original structure of the malleolus ligament, and there is a risk of other ankle joint complications following surgery.Cho et al. [21] reported that the retinaculum reconstruction method used in the clinical treatment of dislocation of the peroneal tendon had a high success rate. Moreover, the recovery time required for post-operative rehabilitation following plaster fixation after superior peroneal retinaculum reconstruction was shorter than that of bone structural changes. The shorter local fixation time contributed to rapid post-operative recovery and early resumption of normal training. However, for this particular population of athletes, single-rivet retinaculum reconstruction may not achieve satisfactory function and strength. As shown in the current study, there were statistically significant differences in the scores between the single-rivet and the multi-rivet fixation methods for post-operative recurrence rates and post-operative ankle function. In this study, 21 patients underwent the multiple-rivet peroneal retinaculum reconstruction (for example, using four rivets to repair the superior peroneal retinaculum). The AOFAS score, VAS score and ankle joint flexion, internal rotation and external rotation angles before and after surgery showed that our surgical procedure effectively and stably repaired the loose or fractured superior peroneal retinaculum, and that the effect of multi-rivet reconstruction was significantly better than that of the single-rivet strategy. All patients who underwent multi-rivet reconstruction had satisfactory stabilization of the peroneal tendon without recurrence during the long-term follow-up period. This suggests that it is necessary to use rivets to reconstruct the superior peroneal retinaculum in Taekwondo athletes and other with requirements for high stability of the ankle joint. The above-mentioned surgical methods make us realize the complexity of the surgical methods for PTD,and surgery is still a primary treatment for athletes with PTD, but it will more or less have an impact on their career after surgical treatment. Some elite taekwondo athletes have lost their careers because of PTD. Therefore, the prevention of the disease from the source has become extremely urgent for the special group.

After we found such a high incidence of PTD in taekwondo athletes in our clinical practice and such a great harm to athletes, we increased our attention to this disease, and also realized the need for prophylactic medication in this special group. Therefore, according to the experience of Traditional Chinese Medicine Osteology, we creatively used astragalus polysaccharide as a Chinese medicine 
monomer to prevent PTD for some athletes at the high risk of dislocation, and achieved satisfactory results in the 4 years of specialized examination and follow-up. Which slowed down the thickening of the peroneal tendon in athletes and also reduced the incidence of PTD. APS, a preventive medicine, may guarantees the athlete's health and prolong their career. A series of experimental results and clinical evidence shows the importance of prophylactic useof APS to reduce the thickening of the peroneal tendon and thus the incidence of PTD in groups such as taekwondo athletes who have high requirements for ankle stability and peroneal tendon.

In the future, the focus should be on more minimally invasive surgical procedures and faster postoperative rehabilitation methods. In addition to this more and more attention will be paid to the preventive treatment of PTD, which will provide protection for more Taekwondo athletes and other similar groups.

\section{Declarations}

\section{Funding}

The authors declare that no funds, grants, or other support were received during the preparation of this manuscript.

\section{Competing Interests}

The authors declare there is no conflicts of interest regarding the publication of this paper.

\section{Author Contribution Statement}

Zhiyu Zhang and Fusheng Yuan conceived and designed research. YunGao, Liqiang Wang and XiaoMa conducted experiments. MengWu contributed new reagents or analytical tools. Bohan Li analyzed data. YunGao, Liqiang Wang and XiaoMa wrote the manuscript. All authors read and approved the manuscript.

Yun Gao, Liqiang Wang and Xiao Ma have contributed equally to this work.

\section{Data availability}

The data set used or analyzed in this study can be obtained from the corresponding author under reasonable request.

\section{Ethics approval}

All methods were carried out in accordance with relevant guidelines and regulations.All animal experiments are conducted in accordance with the "Guidelines for the Care and Use of Laboratory Animals of the National Institutes of Health" and approved by the Institutional Review Committee of the Fourth Hospital of China Medical University.

\section{Consent to participate}


Informed consent was obtained from all individual participants included in the study.

\section{Consent to publish}

Informed consent was obtained from all subjects and/or their legal guardian(s)

\section{References}

[1] R.P. Lystad, H. Pollard, P.L. Graham, Epidemiology of injuries in competition taekwondo: a metaanalysis of observational studies, J Sci Med Sport 12 (2009) 614-621. 10.1016/j.jsams.2008.09.013.

[2] S. Byun, C. An, M. Kim, D. Han, The effects of an exercise program consisting of taekwondo basic movements on posture correction, J Phys Ther Sci 26 (2014) 1585-1588. 10.1589/jpts.26.1585.

[3] M. Ji, Analysis of injuries in taekwondo athletes, J Phys Ther Sci 28 (2016) 231-234.

10.1589/jpts.28.231.

[4] P. Ziai, E. Benca, G. von Skrbensky, A. Graf, F. Wenzel, E. Basad, R. Windhager, T. Buchhorn, The role of the peroneal tendons in passive stabilisation of the ankle joint: an in vitro study, Knee Surg Sports Traumatol Arthrosc 21 (2013) 1404-1408. 10.1007/s00167-012-2273-2.

[5] J. Karlsson, S. Brandsson, P. Kalebo, B.I. Eriksson, Surgical treatment of concomitant chronic ankle instability and longitudinal rupture of the peroneus brevis tendon, Scand J Med Sci Sports 8 (1998) 4249. 10.1111/j.1600-0838.1998.tb00227.x.

[6] A. Nishimura, S. Nakazora, N. Ito, A. Fukuda, K. Kato, A. Sudo, Tendoscopic Double-Row Suture Bridge Peroneal Retinaculum Repair for Recurrent Dislocation of Peroneal Tendons in the Ankle, Arthrosc Tech 5 (2016) e441-446. 10.1016/j.eats.2016.01.028.

[7] W. Miyamoto, M. Takao, K. Yamada, T. Matsushita, Accelerated Versus Traditional Rehabilitation After Anterior Talofibular Ligament Reconstruction for Chronic Lateral Instability of the Ankle in Athletes, Am J Sports Med 42 (2014) 1441-1447. 10.1177/0363546514527418.

[8] K. Davda, K. Malhotra, P. O'Donnell, D. Singh, N. Cullen, Peroneal tendon disorders, EFORT Open Rev 2 (2017) 281-292. 10.1302/2058-5241.2.160047.

[9] K. Winnicki, A. Ochala-Klos, B. Rutowicz, P.A. Pekala, K.A. Tomaszewski, Functional anatomy, histology and biomechanics of the human Achilles tendon - A comprehensive review, Ann Anat 229 (2020) 151461. 10.1016/j.aanat.2020.151461.

[10] A.D. Schoenenberger, J. Foolen, P. Moor, U. Silvan, J.G. Snedeker, Substrate fiber alignment mediates tendon cell response to inflammatory signaling, Acta biomaterialia 71 (2018) 306-317. 10.1016/j.actbio.2018.03.004. 
[11] A. Subramanian, L.F. Kanzaki, J.L. Galloway, T.F. Schilling, Mechanical force regulates tendon extracellular matrix organization and tenocyte morphogenesis through TGFbeta signaling, Elife 7 (2018). 10.7554/eLife.38069.

[12] D. Figueroa, F. Figueroa, R. Calvo, Patellar Tendinopathy: Diagnosis and Treatment, J Am Acad Orthop Surg 24 (2016) e184-e192. 10.5435/JAAOS-D-15-00703.

[13] M. Hammerman, P. Blomgran, A. Dansac, P. Eliasson, P. Aspenberg, Different gene response to mechanical loading during early and late phases of rat Achilles tendon healing, J Appl Physiol (1985) 123 (2017) 800-815. 10.1152/japplphysiol.00323.2017.

[14] L.Z. Rao, Y. Wang, L. Zhang, G. Wu, L. Zhang, F.X. Wang, L.M. Chen, F. Sun, S. Jia, S. Zhang, Q. Yu, J.H. Wei, H.R. Lei, T. Yuan, J. Li, X. Huang, B. Cheng, J. Zhao, Y. Xu, B.W. Mo, C.Y. Wang, H. Zhang, IL-24 deficiency protects mice against bleomycin-induced pulmonary fibrosis by repressing IL-4-induced M2 program in macrophages, Cell Death Differ 28 (2021) 1270-1283. 10.1038/s41418-020-00650-6.

[15] E. Havis, D. Duprez, EGR1 Transcription Factor is a Multifaceted Regulator of Matrix Production in Tendons and Other Connective Tissues, Int J Mol Sci 21 (2020). 10.3390/ijms21051664.

[16] Y.L. Kuo, I.M. Jou, P.H. Wang, T.H. Yang, C.J. Shen, C.C. Hsu, Correlation Between IL-20 and De Quervain's Disease Severity, Ann Plast Surg 82 (2019) S6-S12. 10.1097/SAP.0000000000001701.

[17] C. Dees, S. Potter, Y. Zhang, C. Bergmann, X. Zhou, M. Luber, T. Wohlfahrt, E. Karouzakis, A. Ramming, K. Gelse, A. Yoshimura, R. Jaenisch, O. Distler, G. Schett, J.H. Distler, TGF-beta-induced epigenetic deregulation of SOCS3 facilitates STAT3 signaling to promote fibrosis, J Clin Invest 130 (2020) 23472363. 10.1172/JCl122462.

[18] S.M. Raikin, I. Elias, L.N. Nazarian, Intrasheath subluxation of the peroneal tendons, J Bone Joint Surg Am 90 (2008) 992-999. 10.2106/JBJS.G.00801.

[19] R.E. Boykin, B. Ogunseinde, E.D. McFeely, A. Nasreddine, M.S. Kocher, Preliminary results of calcaneofibular ligament transfer for recurrent peroneal subluxation in children and adolescents, $\mathrm{J}$ Pediatr Orthop 30 (2010) 899-903. 10.1097/BPO.0b013e3181fbfcea.

[20] R. Gaulke, F. Hildebrand, M. Panzica, T. Hufner, C. Krettek, Modified rerouting procedure for failed peroneal tendon dislocation surgery, Clin Orthop Relat Res 468 (2010) 1018-1024. 10.1007/s11999-0090918-3.

[21] J. Cho, J.Y. Kim, D.G. Song, W.C. Lee, Comparison of Outcome After Retinaculum Repair With and Without Fibular Groove Deepening for Recurrent Dislocation of the Peroneal Tendons, Foot Ankle Int 35 (2014) 683-689. 10.1177/1071100714531233.

\section{Figures}




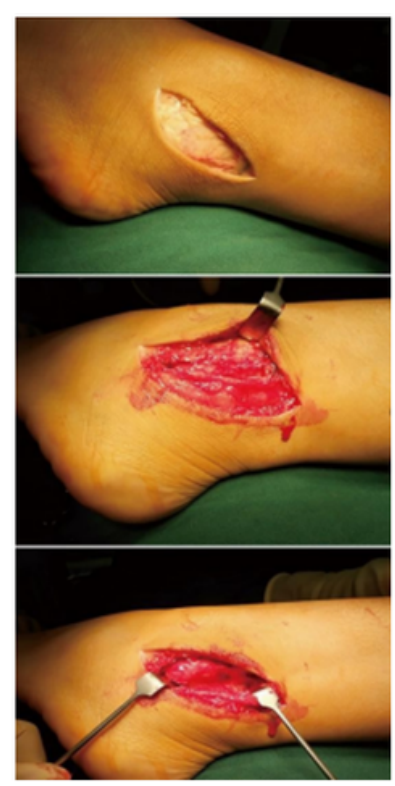

a
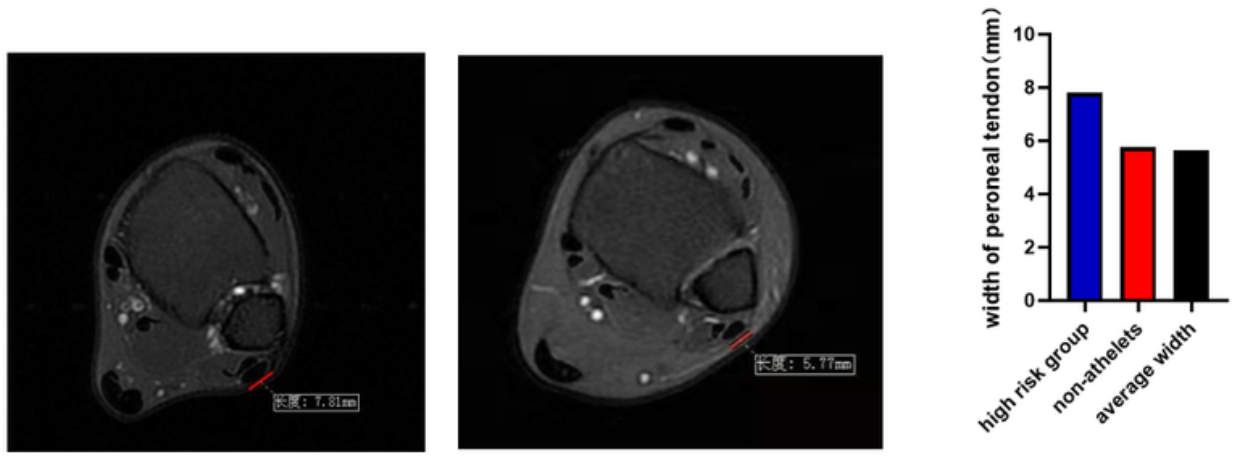

b

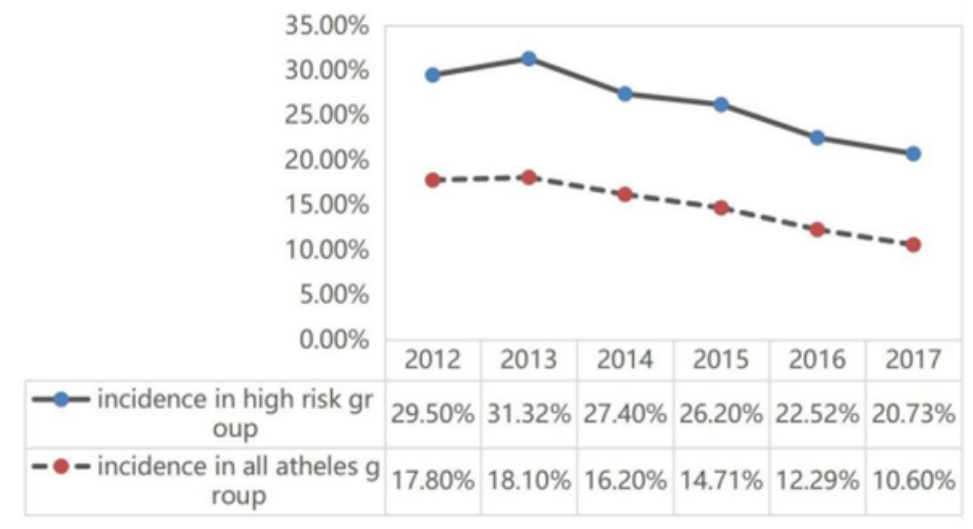

c roup high risk group

$\square$ non-athelets

n average width

\section{Figure 1}

A: intraoperative observation: obviously thickened peroneal tendon and relatively shallow peroneal groove

B: MRI result shows that the thickened peroneal tendon of athlete in high-risk group and normal peroneal tendon of non-athlete group in 220 cases.

C, the incidence of PTD in the high-risk group and all athletes group changed year by year from 2012 to 2017. The abscissa in Figure 1-C is the year, and the ordinate is the incidence. 


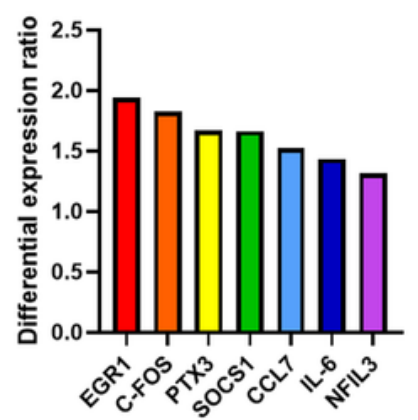

diffrential experission protein in tissue pairs


b
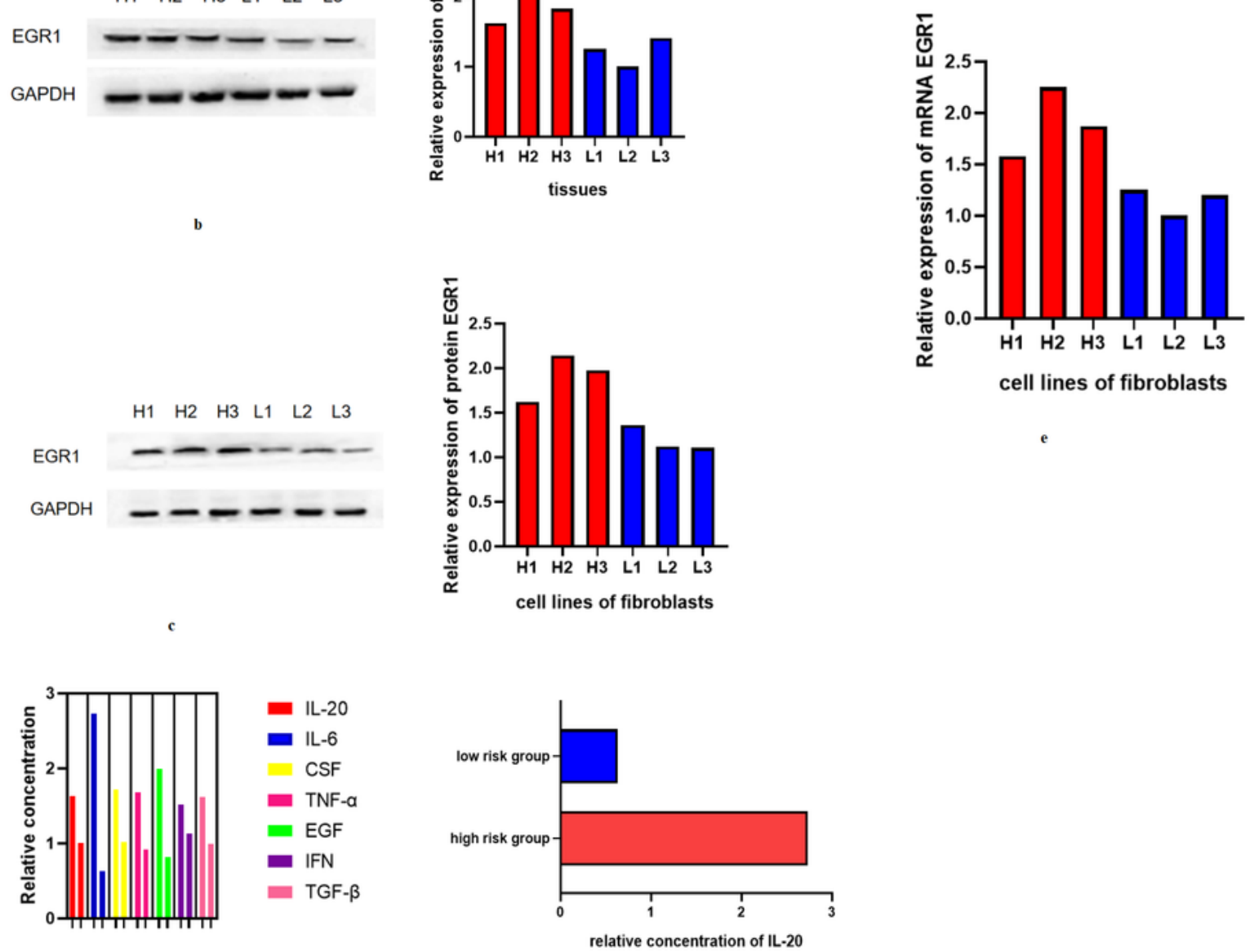

inflammatory cytokines

\section{Figure 2}

A:the top 7 proteins differentially expressed between high-risk and low-risk group(mean expression of high-risk group/mean expression of low-risk group)

B: protein EGR1 extraction and quantification by western blot of tissues from above 6 groups 
Cヌprotein EGR1 extraction and quantification by western blot of fibroblasts from above 6 groupsD: the relative mean expression of protein EGR1 in above 4 groups(expression in tissue and fibroblast).

F:Some typical inflammatory cytokines with obvious concentration differences tested by Elisa kit (each boxes:The histogram on the left represents the high-risk group, and the right-hand represents the low-risk group )and the concentration of IL-20 between the high-risk and low-risk groups are quantified
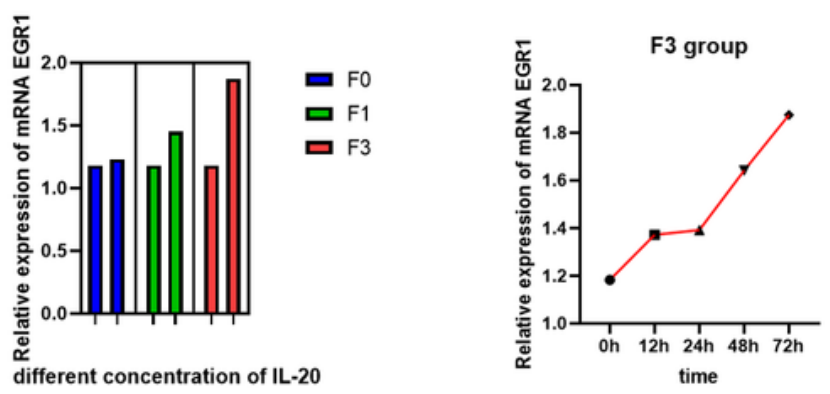

\section{a}
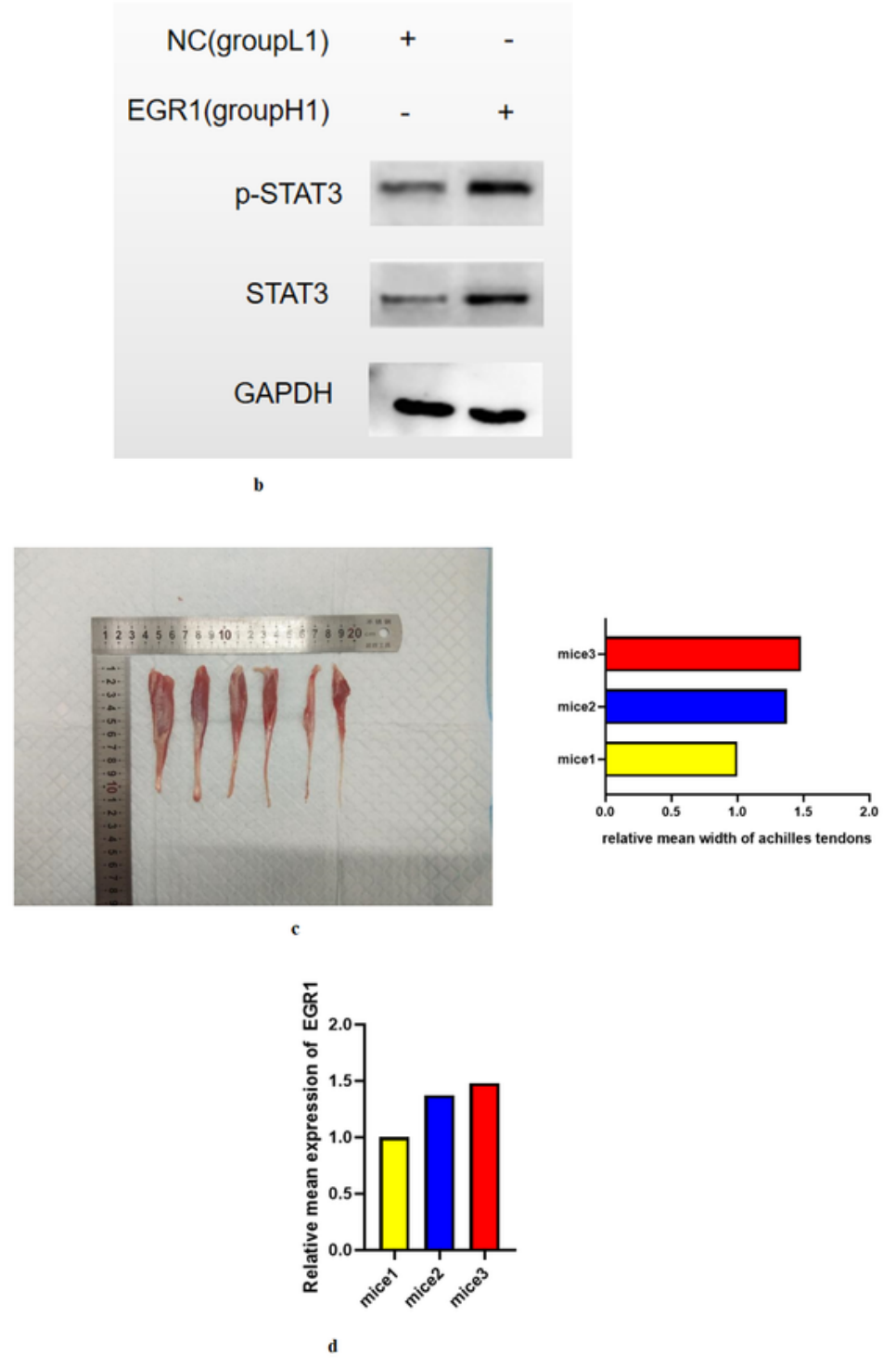


\section{Figure 3}

A:qRTPCR specifically quantified the expression of EGR1 in fibroblasts cultured for 72 hours at different concentration of IL-20. (In each box, the histogram on the left represents the expression of EGR1 at 0h, and the right represents the expression of EGR1 at 72h) and the relative expression of EGR1 at different times in the F3 group.

B:Activation of STAT3 pathway by cell lines of $\mathrm{H} 1$ group (ie the relatively high expression group of EGR1) and L1 group (ie the relatively low expression group of EGR1)

C:Take out the bilateral Achilles tendons of mice 1, 2 and 3 ( 45 days after the injection). The visual diagram is as shown in the figure,and the average value of the relative width in both Achilles tendons from mice1, 2, 3 (mice3 is the two on the left)

D:the quantification of the average expression of EGR1 in both Achilles tendons from mice1,2,3 

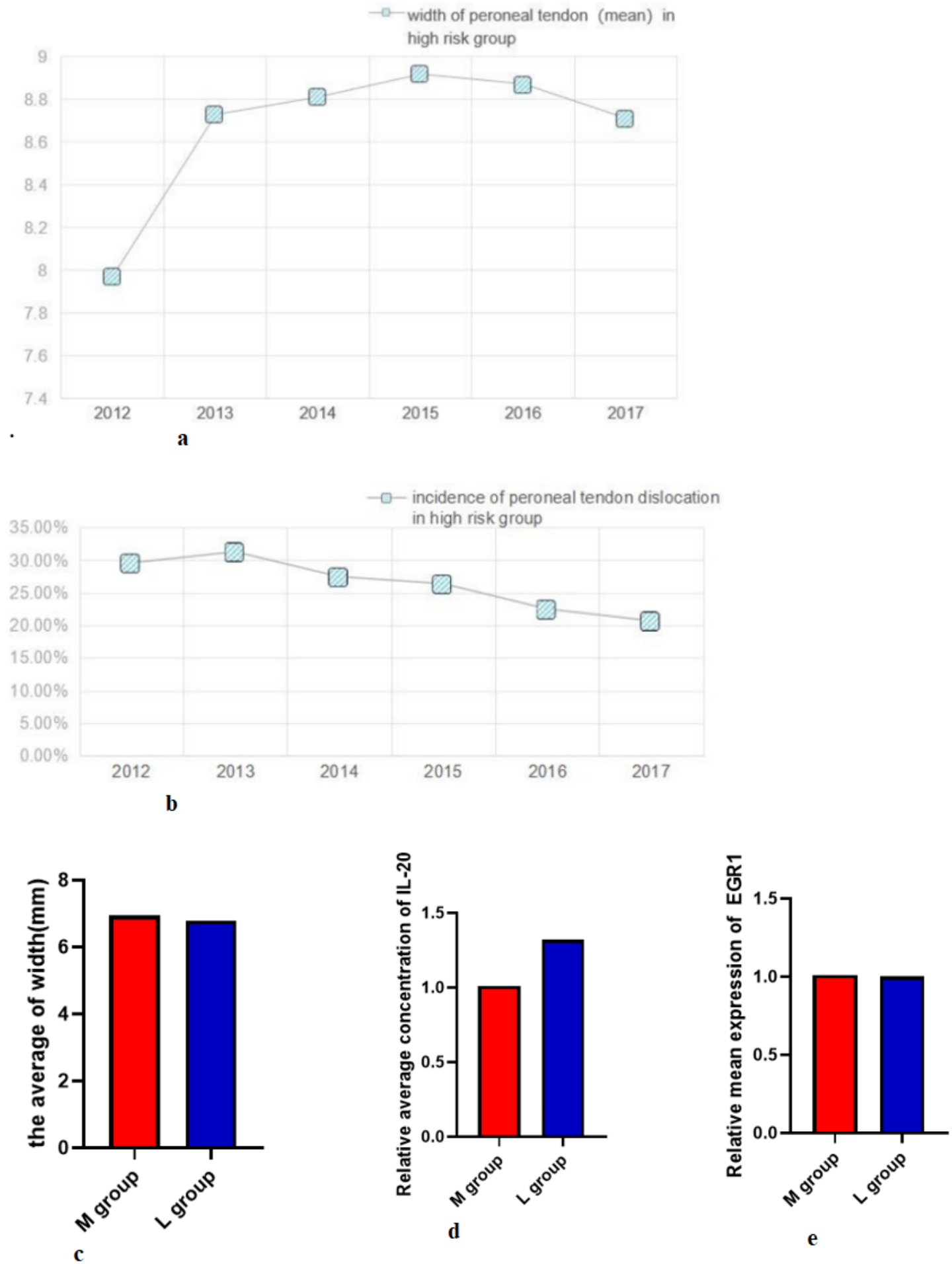

\section{Figure 4}

A, year-on-year changes in the mean peroneal tendon width of athletes in the high-risk group from 2012 to 2017. The abscissa of Figure 4-A is the year, and the ordinate is the width of the peroneal tendon (the unit is $\mathrm{mm}$ ) 
$B$, year-by-year variation in the incidence of PTD in athletes in the high-risk group from 2012 to 2017. The abscissa of Figure 4-B is the year, and the ordinate is the incidence of PTD in high-risk group

C:the average of width between $\mathrm{M}$ group and $\mathrm{L}$ group

D:the relative concentration of IL-20 between $\mathrm{M}$ group and $\mathrm{L}$ group

E:the relative average expression of EGR1 between $M$ group and $L$ group 